\title{
Population dynamics of sporogony for Plasmodium vivax parasites from western Thailand developing within three species of colonized Anopheles mosquitoes
}

\author{
Gabriela E Zollner ${ }^{1}$, Narong Ponsa ${ }^{2}$, Gabriel W Garman ${ }^{3}$, \\ Shreekanta Poudel ${ }^{3}$, Jeffrey A Bell ${ }^{2}$, Jetsumon Sattabongkot ${ }^{2}$, \\ Russell E Coleman ${ }^{1}$ and Jefferson A Vaughan*3
}

\begin{abstract}
Address: ${ }^{1}$ Department of Entomology, Walter Reed Army Institute of Research, Silver Spring, MD 20910-7500, USA, ${ }^{2}$ Department of Entomology, USAMC-AFRIMS, Bangkok, Thailand and '32Department of Biology, University of North Dakota, Grand Forks, ND 58202-9019, USA

Email: Gabriela E Zollner - Gabriela.Zollner@na.amedd.army.mil; Narong Ponsa - NarongP@AFRIMS.ORG;

Gabriel W Garman - Gabriel.Garman@und.nodak.edu; Shreekanta Poudel - Shreekanta.Poudel@und.nodak.edu;

Jeffrey A Bell - piciformes@yahoo.com; Jetsumon Sattabongkot - JetsumonP@afrims.org;

Russell E Coleman - Russell.Coleman@NA.AMEDD.ARMY.MIL; Jefferson A Vaughan* - jefferson_vaughan@und.nodak.edu

* Corresponding author
\end{abstract}

Published: 03 August 2006

Malaria Journal 2006, 5:68 doi:10.1186/1475-2875-5-68

This article is available from: http://www.malariajournal.com/content/5///68

(C) 2006 Zollner et al; licensee BioMed Central Ltd.

This is an Open Access article distributed under the terms of the Creative Commons Attribution License (http://creativecommons.org/licenses/by/2.0), which permits unrestricted use, distribution, and reproduction in any medium, provided the original work is properly cited.
Received: 05 October 2005

Accepted: 03 August 2006

\begin{abstract}
Background: The population dynamics of Plasmodium sporogony within mosquitoes consists of an early phase where parasite abundance decreases during the transition from gametocyte to oocyst, an intermediate phase where parasite abundance remains static as oocysts, and a later phase where parasite abundance increases during the release of progeny sporozoites from oocysts. Sporogonic development is complete when sporozoites invade the mosquito salivary glands. The dynamics and efficiency of this developmental sequence were determined in laboratory strains of Anopheles dirus, Anopheles minimus and Anopheles sawadwongporni mosquitoes for Plasmodium vivax parasites circulating naturally in western Thailand.
\end{abstract}

Methods: Mosquitoes were fed blood from 20 symptomatic Thai adults via membrane feeders. Absolute densities were estimated for macrogametocytes, round stages (= female gametes/zygotes), ookinetes, oocysts, haemolymph sporozoites and salivary gland sporozoites. From these census data, five aspects of population dynamics were analysed; I) changes in life-stage prevalence during early sporogony, 2) kinetics of life-stage formation, 3) efficiency of life-stage transitions, 4) density relationships between successive life-stages, and 5) parasite aggregation patterns.

Results: There was no difference among the three mosquito species tested in total losses incurred by $P$. vivax populations during early sporogony. Averaged across all infections, parasite populations incurred a 68-fold loss in abundance, with losses of ca. 19-fold, 2-fold and 2-fold at the first (= gametogenesis/fertilization), second (= round stage transformation), and third (= ookinete migration) life-stage transitions, respectively. However, total losses varied widely among infections, ranging from 6-fold to over 2,000-fold loss. Losses during gametogenesis/ fertilization accounted for most of this variability, indicating that gametocytes originating from some volunteers were more fertile than those from other volunteers. Although reasons for such variability were not determined, gametocyte fertility was not correlated with blood haematocrit, asexual parasitaemia, gametocyte density or gametocyte sex ratio. Round stages and ookinetes were present in mosquito midguts for up to 48 hours and development was asynchronous. Parasite losses during fertilization and round stage differentiation were more 
influenced by factors intrinsic to the parasite and/or factors in the blood, whereas ookinete losses were more strongly influenced by mosquito factors. Oocysts released sporozoites on days 12 to 14 , but even by day 22 many oocysts were still present on the midgut. The per capita production was estimated to be approximately 500 sporozoites per oocyst and approximately $75 \%$ of the sporozoites released into the haemocoel successfully invaded the salivary glands.

Conclusion: The major developmental bottleneck in early sporogony occurred during the transition from macrogametocyte to round stage. Sporozoite invasion into the salivary glands was very efficient. Information on the natural population dynamics of sporogony within malaria-endemic areas may benefit intervention strategies that target early sporogony (e.g., transmission blocking vaccines, transgenic mosquitoes).

\section{Background}

Transmission of malaria relies on the successful development of Plasmodium parasites within mosquitoes, a process termed sporogony. Sporogony is a complex event involving several morphologically distinct life-stages $[1,2]$ and begins when mosquitoes ingest blood containing male and female gametocytes. Sporogony has three basic phases based on changes that occur in parasite abundance within the mosquito vector. The first phase may be termed "early sporogony", a relatively brief period of time where parasites numbers typically decrease within the mosquito. Early events include gametogenesis and fertilization, zygote transformation into ookinetes, ookinete motility through the bloodmeal and peritrophic matrix, penetration across midgut epithelia, and encystment beneath the midgut basal lamina to form oocysts. These events occur during the time that the engorged mosquito is digesting its bloodmeal ( $\mathrm{ca} .2$ days). Early sporogony is followed by a period lasting up to a week or more (= "mid-sporogony") where parasites are in the oocyst stage. Oocysts grow in size but their numbers remain static. The enlarging oocysts undergo multiple rounds of mitosis to form a syncytium, followed by cellular differentiation to form several thousand daughter cells (= sporozoites). The final phase is "late sporogony" which involves release of the sporozoites into the mosquito haemocoel and their subsequent invasion into the mosquito salivary glands. Sporogony is considered complete after sporozoites successfully infect the mosquito salivary glands (ca. 10 to 16 days after initiation) and mosquitoes are able to transmit the parasite to a vertebrate host by infectious bite.

Not every mosquito species supports sporogony of every Plasmodium species and disruptions at any point along the developmental sequence diminish the ability of a given mosquito species to transmit malaria. The notion that it is possible to reduce malaria transmission by disrupting sporogony in nature via transmission blocking vaccines [3-5] or introduction of refractory genes into vector populations [6-8] has generated new findings that have greatly increased our understanding of the cellular and molecular details of sporogony [9-11]. Sporogony has been less studied in terms of its population dynamics - i.e., quanti- fying the successive changes in parasite abundance and distribution throughout the developmental sequence [1221]. When mosquitoes feed on a gametocytaemic person, a portion of the gametocyte population within that person becomes distributed into discrete "sub-populations" (i.e., mosquitoes), somewhat analogous in concept to that of a meta-population. Unless the mosquito feeds on another gametocytaemic person, there is no immigration or emigration of parasites into or out of the mosquito until sporogony is complete. If absolute densities of the various parasite life-stages can be quantified within a mosquito, then the overall efficiency and dynamics of sporogony can be described. While it is virtually impossible to monitor the parasites developing within a single mosquito, the population dynamics of a parasite metapopulation can be monitored by sampling many mosquitoes over time provided that all the parasites within a cohort of mosquitoes originated from the same progenitor population (i.e., the same infected person).

Comparing the population dynamics of different parasite populations and parasite species developing within different vector species can reveal the relative efficiencies of the various life-stage transitions. Furthermore, knowledge of transitional efficiencies within naturally-occurring human/Anopheles transmission systems can be combined with knowledge of the cellular/molecular processes of sporogony and mosquito immunity to identify which of these processes are the most crucial in regulating plasmodial sporogony in nature. The natural efficiency of sporogony for Plasmodium species infecting humans has been described only for the early phase of Plasmodium falciparum sporogony in Anopheles gambiae mosquitoes at a few localities in tropical Africa [17-21]. This report describes the population dynamics of sporogony for natural Plasmodium vivax infections from western Thailand in three indigenous species of colonized Anopheles mosquito vectors.

\section{Methods \\ Mosquitoes}

Three species of colonized Anopheles vectors were used in this study; Anopheles dirus sensu stricto (= dirus complex), 
Anopheles minimus A (= minimus complex), and Anopheles sawadwongporni (= maculatus complex). These species have been maintained in colony at the Armed Forces Research Institute of Medical Sciences (AFRIMS) in Bangkok, Thailand, for $>25,14$, and 9 years, respectively. All three species are important vectors of P. vivax in Thailand [22].

\section{Volunteers}

Human subjects involved in this study were comprised of 20 adult ( $\geq 18$ yrs) volunteers seeking treatment for uncomplicated malaria at Mae Sot and Mae Kasa clinics, Tak Province in northwestern Thailand. Parasites from 15 of the volunteers were used to estimate the efficiency of early sporogony (i.e., gametocyte to oocyst stages). Parasites from five of the volunteers were used to estimate the efficiency of late sporogony (i.e., sporozoite production and invasion into salivary glands). Prior to conducting this research, the study protocol received approval from the Institutional Ethics Committee of the Thai Ministry of Public Health and the Human Subjects Research Review Boards of the Walter Reed Army Institute of Research and the University of North Dakota. To diagnose for malaria, thick and thin blood smears were prepared from each volunteer by the clinic staff, stained with $10 \%$ Giemsa and examined for malarial parasites. If $P$. vivax gametocytes were present and the volunteers met specific criteria outlined in the approved human subjects use protocols, the volunteers were asked to enroll in the study and complete informed consent forms. Approximately $10 \mathrm{ml}$ of blood was collected by venipuncture and placed in a $37^{\circ} \mathrm{C}$ water bath. Additional blood samples (fingerprick) were blotted onto strips of filter paper to distinguish the P. vivax VK210 and VK247 strains, using nested PCR methods described previously [23]. Volunteers received antimalarial treatment from the clinic staff and were released. Blood smears were later examined more thoroughly (100 microscopic fields at $1,000 \times$ oil immersion) to quantify leukocyte densities, asexual and sexual stage parasite densities and gametocyte sex ratios. In this paper, the term "macrogametocyte" is used to describe the female gametocyte detected in peripheral blood smears.

\section{Infecting mosquitoes}

Five to seven day old, con-specific, nulliparous female mosquitoes in cylindrical cardboard containers were transported by automobile from AFRIMS to Mae Sot clinic. Mosquitoes were deprived of sucrose overnight to enhance their willingness to feed. One milliliter of heparinized blood from each volunteer was added to a 5$\mathrm{cm}$ diameter water-jacketed glass membrane feeder fitted with a Baudruche membrane. Blood was kept at a constant $37^{\circ} \mathrm{C}$ during the mosquito feeding to attract the mosquitoes and prevent premature gametogenesis. Mosquitoes were allowed to feed for 30 minutes and unfed mosquitoes were removed. Engorged mosquitoes were maintained in an insectary at $24^{\circ} \mathrm{C}$ and ambient humidity for 2 days at the Mae Sot clinic during which time round stages and ookinetes were sampled. In this paper, the term "round stages" is used to describe both unfertilized female gametes (i.e., macrogametes) and fertilized zygotes. Mosquitoes were then transported back to the AFRIMS insectary and maintained at $24^{\circ} \mathrm{C}$ for up to 22 days for oocyst and sporozoite sampling.

\section{Early sporogony - macrogametocyte sampling}

Indirect estimates of macrogametocyte densities per mosquito were obtained using methods similar to those described previously [12]. Macrogametocyte densities per 100 leukocytes were determined for each volunteer from his/her thick blood film. These values were then multiplied by the corresponding leukocyte densities within the mosquito bloodmeals to obtain the estimated density of macrogametocytes in the mosquito bloodmeal. To estimate the average leukocyte densities of the mosquito bloodmeals, the mean volumes of blood ingested by each mosquito species were determined by weighing pools of unfed mosquitoes and pools of mosquitoes immediately after feeding on uninfected blood. The differences in weight between unfed and fed mosquitoes indicated the amount of blood retained in the bloodmeal. A leukocyte density of 7,015 leukocytes per $\mu \mathrm{l}$ blood was used throughout to calculate leukocyte densities. This value was obtained by performing leukocyte counts on 5 of the volunteers and averaging the individual counts. For example, if a microscopic examination of a volunteer's thick smear yielded 10 macrogametocytes per 100 leukocytes and the mean volume of blood ingested by A. dirus mosquitoes was $1.5 \mu \mathrm{l}$, then the theoretical number of gametocytes ingested would be 10 divided by $100 \quad(=0.10$ macrogametocytes per leukocyte) times 7,015 leukocytes per $\mu \mathrm{l}$ times $1.5 \mu \mathrm{l}$ blood ingested by $A$. dirus; the product of which yields 1,052 macrogametocytes per mosquito bloodmeal for that hypothetical case.

\section{Early sporogony - round stage and ookinete sampling and bloodmeal digestion kinetics}

Bloodmeals were examined for round stages and ookinetes using immunofluorescent antibody staining techniques described previously [24]. Groups of mosquitoes were dissected at $12,18,20,22,24,26,36$ or $48 \mathrm{~h}$ after feeding. The average numbers of mosquitoes dissected per sampling interval for A. dirus, A. minimus and A. sawadwongporni were 12, 6, and 6 mosquitoes, respectively. Fluorescing parasites were counted using $400 \mathrm{X}$ epifluorescent microscopy and classified as either round stages, retort ookinetes (Stages II or III) or mature ookinetes (Stages IVVI combined) [25] (Figure 1). The kinetics of mosquito bloodmeal digestion was also examined because early sporogony occurs within the context of bloodmeal digestion. A. dirus and A. sawadwongporni mosquitoes were fed 


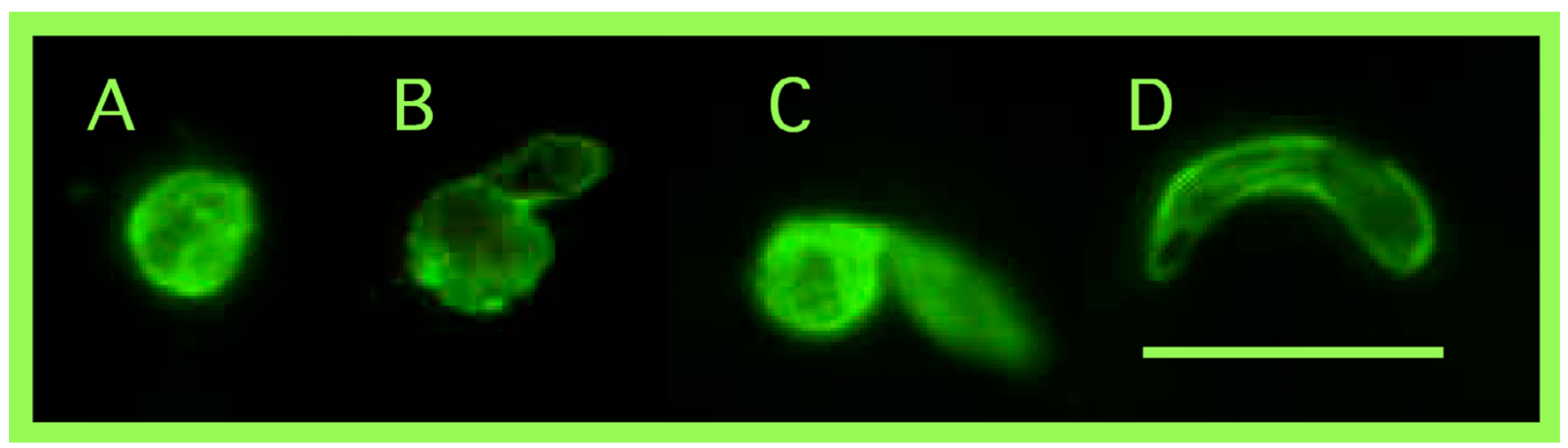

Figure I

Early sporogonic life-stages of Plasmodium vivax developing within the blood meal of Anopheles mosquitoes. Parasites are visualized by immunostaining with monoclonal antibody specific against the $25 \mathrm{kD}$ protein of sexual stages of $P$. vivax. A. Round stage representing either female gamete or zygote; B. stage II retort ookinete; C. stage III retort ookinete; D. stage IV-VI mature ookinete.

heparinized, uninfected human blood via membrane feeding and maintained at $24^{\circ} \mathrm{C}$. Bloodmeal volumes were determined as described above. Groups of mosquitoes were dissected at $0,12,24,30,36$ and 48 hours after feeding and individual bloodmeals were diluted 1:200 in buffered saline. Erythrocytes were counted using a haemocytometer and appropriate calculations were made to determine the mean erythrocyte densities of mosquito bloodmeals at each sampling interval.

\section{Oocyst sampling}

Oocysts were counted at various intervals, beginning at 7 to 22 days after infectious feeds. Mosquito midguts were individually excised, placed in a droplet of diluted mercurochrome on a glass slide, compressed with a coverslip and examined at 100-400X bright field microscopy. Oocysts were measured with an ocular micrometer at 400X.

\section{Late sporogony - sporozoite sampling}

For this aspect of the study, only infections that contained high oocyst prevalences were used because the sampling techniques involved were quite meticulous and laborious. From day 7 to 22 after the infectious bloodmeal, groups of 8 to 15 mosquitoes each were removed every other day and processed in such a manner that allowed estimation of the absolute densities of oocysts, haemolymph sporozoites and salivary gland sporozoites within single mosquitoes [12]. First, haemolymph was collected by haemocoel perfusion. The mosquito was immobilized by chilling and restrained by light vacuum drawn through the open end of a bent syringe needle. A small incision was made near the posterior end of the abdomen. A finetipped glass needle mounted in a micromanipulator (World Precision Instruments, Sarasota, FL) and connected by tubing to a $500 \mathrm{cc}$ syringe (= hand vacuum/ pump) was filled with ca. $15 \mu \mathrm{l}$ of RPMI media and inserted into the thorax of the restrained mosquito. The haemocoel was flushed by gently pressing the plunger of the syringe. The perfusate exuding from the abdominal incision was collected with a micro-capillary tube and 10 $\mu \mathrm{l}$ was loaded onto a haemocytometer (= haemolymph sporozoite sample). Next, the paired salivary glands were excised and transferred with a fine minuten hook into a glass micro-grinder (Kontes Glass Co., Vineland, NJ) containing $35 \mu \mathrm{l}$ of RPMI media. Gland pairs were triturated and $10 \mu \mathrm{l}$ of the triturate was loaded onto the opposite side of the same haemocytometer (= salivary gland sporozoite sample). Both samples were examined at $400 \mathrm{x}$ phase-constrast and the sporozoites were counted. Finally, the midgut was dissected and examined for oocysts as described above. Mosquitoes were processed in this manner until a minimum of five infected mosquitoes had been detected for each sampling interval. Only infected mosquitoes were included in data analyses.

\section{Data analysis}

Five aspects of population dynamics were analysed; 1) stage-specific prevalence during early sporogony, 2) stagespecific kinetics of early and late sporogony, 3 ) estimation of absolute densities and transitional efficiencies between life-stages (i.e., cohort life tables), 4) density relationships between life-stages, and 5) aggregation patterns during early sporogony. Chi square analyses were used to compare the prevalences of early sporogonic life-stages among volunteer and mosquito species. Count data on life-stage densities were first tested for normality (Shapiro-Wilk Normality Tests) and the overall effects of independent variables (e.g., mosquito species, volunteer number, haematocrit, etc.) on mean life-stage densities were tested using analysis of variance (ANOVA) for normally distributed variables and Kruskal-Wallis ANOVA for non-nor- 
mally distributed variables. Kinetics of life-stage formation were determined for each infection by plotting over time the mean densities of round stages, and ookinetes (Stages II to VI combined) for early sporogony and the mean densities of oocysts, haemolymph sporozoites and salivary gland sporozoites for late sporogony. When this was done, it was evident that parasite life-stage development was typically asynchronous, with multiple peaks in abundance that overlapped with successive life-stages. To construct cohort life tables, it was necessary to estimate the absolute density entering each life-stage. Estimating density was straightforward for macrogametocytes and oocysts because there was no overlap in recruitment from previous life-stages. Macrogametocyte densities were based on counts from a blood smear, whereas oocysts remained the only life-stage present in mosquitoes for at least a week. However with the more dynamic life-stages (i.e., round stages, ookinetes, and haemolymph sporozoites), choosing a single time interval on which to base "peak estimates" for the absolute density of a life-stage was more problematic due to overlapping stage-specific recruitment and multiple peaks in abundance. The ecological literature describes different methods for estimating the numbers entering a life-stage from a series of population samples and each method has its own specific assumptions and requirements [26]. One method that has compatible assumptions with sporogony is to plot lifestage densities over time and compute the area under the curve. Unfortunately, the sampling intervals resulted in truncated density curves (i.e., parasite sampling did not begin soon enough or continue long enough to have defined endpoints). Without the tail ends of the curve, the "integration-under-the curve" method yielded inflated estimates of density (data not shown). Instead, a simple ranking system was devised to define the "peak" life-stage densities with which to construct cohort life tables. For each infection and mosquito species, the count data for round stages, ookinetes and sporozoites were ranked from highest to lowest and the arithmetic mean for each data column was designated as the threshold or "cut-off" value. Counts equaling or exceeding the mean were then averaged and the resulting values were used thereafter to represent the "peak" densities for each life-stage within an infection and mosquito species. Counts below the threshold were not used to estimate "peak" life-stage densities. The advantage of this threshold method is that ranking all parasite counts disregarded the time intervals from which a sample was collected. This eliminated temporal variation in the waxing and waning of populations among infections, as well as any developmental asynchrony and bimodal peaks in abundance. A potential disadvantage of this method is that individual mosquitoes within a cohort with inherently low peak densities may have been underrepresented in the construction of life tables. However, this may be mitigated somewhat, because any potential under-representation was applied evenly across all lifestages (except oocysts) within a cohort. Furthermore, multiple parasite cohorts (i.e., infections) consisting of a wide range of starting parasite densities were sampled. Once peak estimates of life-stage densities were obtained using the described "threshold ranking" system, the transitional efficiencies of early sporogonic life-stages were calculated using the population mortality coefficient, $k$, or "killing power", which is simply the difference between the peak densities of 2 consecutive life-stages expressed as logarithms [27]. The first major life-stage transition is the macrogametocyte-to-round stage transition, or $k-1$. Thus, $k-1=$ $\log _{10}$ (macrogametocyte) minus $\log _{10}$ (round stage) and represents the intensity of parasite losses during female gametogenesis and/or fertilization. The second major transition is the round stage-to-ookinete transition, or $k-2$. Thus, $k-2=\log _{10}$ (round stage) minus $\log _{10}$ (ookinete) and represents the intensity of parasite losses during round stage transformation to ookinetes. The third major transition is the ookinete-to-oocyst transition, or $k-3$. Thus, $k-3$ $=\log _{10}$ (ookinete) minus $\log _{10}$ (oocyst) and represents the intensity of parasite losses due to ookinetes crossing the midgut and forming oocysts. The total mortality from macrogametocyte to oocyst, or $K$, was calculated by summing the individual k-values $(=k-1+k-2+k-3)$. In instances, where a negative $k$-value was computed - signifying a net gain in numbers - the value was set to zero because macrogametocytes, round stages and ookinetes are non-replicating life-stages. Mortality coefficients are based on logarithms and thus are not necessarily intuitive to everyone. To make them more intuitive, parasite mortalities can also be expressed as the antilog of $k$ values (= "fold loss") or as a "percentage loss", using the formula 100 100 (1/antilog k) [17]. Approximately 60 to 70 mosquitoes of each species were required from each infectious feed to acquire a complete life table (i.e., macrogametocyte, round stage, ookinete and oocyst density estimates). Unfortunately, colony production and feeding success differed among the 3 mosquito species and hence, the numbers of complete life tables available for analyses differed among mosquito species. Due to its superior productivity in colony and willingness to engorge from a membrane feeder, $A$. dirus mosquitoes yielded complete life tables from all 15 volunteers. A. minimus and A. sawadwongporni yielded seven and three complete life tables, respectively, and nine and seven partial life tables (i.e., macrogametocyte, round stage and ookinete estimates only), respectively. Of the 15 volunteer feeds, 10 were "co-infections" - i.e., blood from the same volunteer was fed to more than one species of mosquito. Life-stage densities and mortality coefficients from paired data (i.e., co-infections) were compared using paired t-tests or Wilcoxon-signed rank tests, depending on whether or not data were normally distributed. Linear regressions were used to determine density relationships among successive life-stages and life- 
stage mortalities. Significances of density relationships were tested using F-tests. For each infection, stage-specific parasite populations during early sporogony were examined to determine whether they had regular, random or dispersed (i.e., aggregated) distributions among mosquitoes. The degree of aggregation or dispersion displayed by a parasite population is indicative of the degree of heterogeneity in mosquito-to-mosquito susceptibility to infection. Green's index of dispersion [28] was computed on entire, unranked data for each infection having more than one infected mosquito per life-stage. Indices of dispersion could not be computed for macrogametocyte populations because macrogametocyte density estimates were obtained indirectly (see above) and did not have sample variances associated with them. Green's index was calculated as: $\left(s^{2} / m-1\right) /\left(\sum x-1\right)$ where $s^{2}=$ variance, $m=$ mean, $\Sigma x=$ sum. Values can range from $-1 / \Sigma(x-1)$ indicative of a totally uniform distribution, ' $O$ ' indicative of a totally random distribution to ' 1 ' indicative of perfect clumping. Green's index is independent of changes in the sample mean and sample size, making it appropriate for comparing different populations that vary in these parameters [29]. Paired t-tests were used to determine significant differences in overall Green's indices for life-stages. A 0.05 level of significance was used for all statistical tests (Statistix v. 8, Tallahassee, FL).

\section{Results}

\section{Data set}

For studies on early sporogony, the number of infections for A. dirus, A. minimus and A. sawadwongporni were 15, 9, and 7 respectively. A total of 3,238 mosquitoes were processed - 1,655 mosquitoes (1,057 A. dirus, 402 A. minimus and $196 \mathrm{~A}$. sawadwongporni) for round stages and ookinetes and 1,583 mosquitoes $(1,423 \mathrm{~A}$. dirus, $126 \mathrm{~A}$. minimus and 34 A. sawadwongporni) for oocysts. A total of 37,421 parasites were counted - 428 gametocytes, 4,379 asexual blood stages, 11,786 round stages, 10,319 ookinetes, and 10,509 oocysts. For studies on late sporogony, the number of infections for $A$. dirus, and A. minimus were 5 and 2 , respectively. Infections with $A$. sawadwongporni failed. A total of 514 mosquitoes (394 A. dirus and $120 \mathrm{~A}$. minimus) were processed. A total of 41,212 parasites were counted - 13,123 oocysts, 14,693 haemocoel sporozoites, and 13,396 salivary gland sporozoites. Sporozoite count data were haemocytometer counts (i.e., subsample counts), whereas round stage, ookinete and oocyst count data were direct counts.

\section{Early sporogony - volunteer infectiousness}

Fourteen volunteers were infected with the VK210 strain of $P$. vivax, and one volunteer (Volunteer 3) was infected with the VK247 strain of $P$. vivax. All were symptomatic for malaria and displayed a range of haematocrits (32 to $54 \%$; mean $=43 \pm 2 \%$ ), asexual parasitaemias (543 to
39,647 trophozoites per $\mu \mathrm{l}$; mean $=5,552 \pm 2,540$ ), gametocytaemias ( 65 to 3,632 gametocytes per $\mu \mathrm{l}$; mean $=540$ \pm 230 ) and female-to-male gametocyte ratios (0.5 to 6.1; mean $=2.7 \pm 0.5$ ). However, none of these parameters correlated with the infectiousness of volunteers to mosquitoes (i.e., oocyst density or prevalence; linear regressions, $\mathrm{p}>0.05)$. Volunteers were classified retrospectively into 2 groups; HIGH (10 volunteers who at the time of donating blood, would likely perpetuate transmission in nature [i.e., volunteers 1, 2, 3, 5, 6, 11, 12, 13, 14, 15]) and LOW (five volunteers who were unlikely to perpetuate transmission in nature [i.e., volunteers $4,7,8,9,10])$. The criterion for this classification was whether or not the volunteer yielded a geometric mean of $\geq 1$ oocyst per midgut and $\geq 30 \%$ oocyst infection prevalence in $A$. dirus. In general, this classification held up for the two other mosquito species with the exception that three of the 10 volunteers classified as HIGH (i.e., volunteers 12, 13, 14) produced geometric means of less than 1 oocyst per midgut in $A$. minimus and A. sawadwongporni.

\section{Early sporogony - bloodmeal size, kinetics of erythrocyte digestion and ookinete formation}

Mean bloodmeal weights for A. dirus, A. minimus and A. sawadwongporni feeding on membrane feeders were $1.53 \pm$ $0.42,0.60 \pm 0.27$, and $0.64 \pm 0.04 \mathrm{mg}$, respectively. There was a lag time of at least 12 (A. sawadwongporni) to 24 hours (A. dirus) before substantial digestion of erythrocytes occurred (data not shown). During this lag time, bloodmeals clotted and peritrophic matrices formed. Between 30 and 48 hours, digestion of erythrocytes accelerated and many erythrocytes appeared visibly damaged. By 48 hours, most of the erythrocytes were digested. Round stages and ookinetes were present in mosquito bloodmeals from 12 to 48 hours after ingestion of gametocytes. Developmental kinetics of these life-stages was asynchronous and there was considerable overlap in round stage and ookinete abundances. Although patterns varied among infections and mosquito species, 3 general patterns were observed; a single peak density (Figure 2A), a bimodal peak (Figure 2B) and a broad, extended peak (Figure 2C). There were no obvious trends in these patterns among mosquito species and no one pattern predominated. When all infections and mosquito species were combined, round stages were considerably more prevalent than ookinetes at 12 hours ( $81 \%$ versus $19 \%$, respectively), but less so at 18 hours (58\% versus $42 \%$ ) and later (Table 1). This suggests that many round stages transformed to ookinetes between 12 and 18 hours. Retort-form ookinetes (Stages II and III, see Figure 1) were neither abundant nor prevalent during the entire sampling period, which suggested that these stages were ephemeral and developed rapidly into mature forms. Surprisingly, both round stages and ookinetes were still plen- 


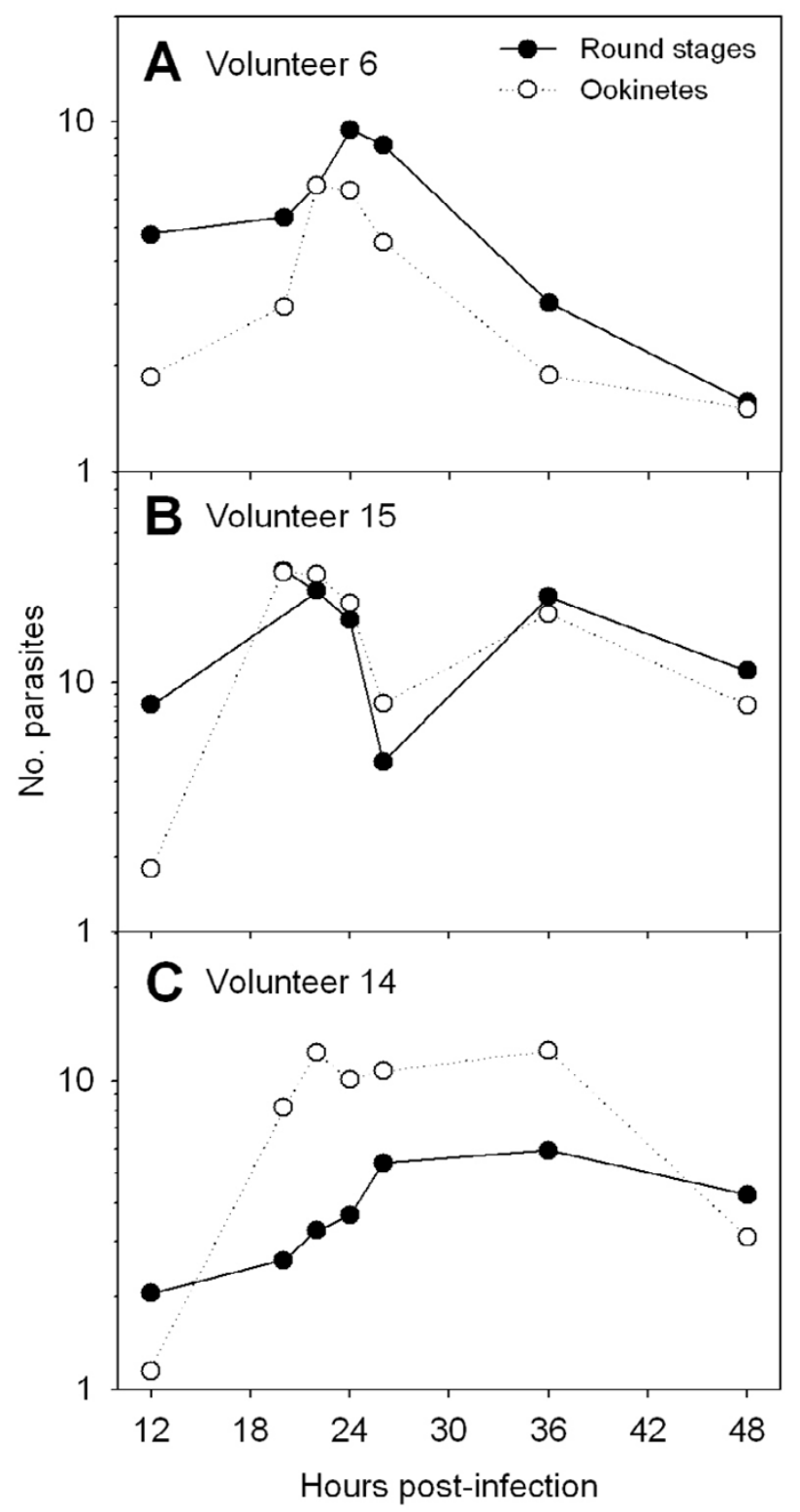

Figure 2

Developmental kinetics of Plasmodium vivax round stage and ookinete life-stages in Anopheles dirus mosquitoes. These figures show different patterns of ookinete development observed for individual infections and are representative of patterns observed for 31 separate infections in A. dirus, A. minimus and $A$. sawadwongporni mosquitoes.

tiful in mosquito midguts at 48 hours (Table 1, Figure 2), after most of the erythrocytes had been digested.

\section{Early sporogony - life-stage prevalences among mosquitoes}

A high proportion of bloodmeals from mosquitoes fed on the HIGH category of volunteers contained round stages $(>88 \%)$ and mature ookinetes $(>75 \%)$, whereas a substantially lower proportion of bloodmeals from mosquitoes fed on the LOW category of volunteers contained round stages $(<30 \%)$ and mature ookinetes $(<13 \%)$ (Table 2). The composite age-class (i.e., round stages and ookinetes combined) was comprised of a significantly greater proportion of round stages in the LOW category ( $94 \%$ of the total 1,426 parasites counted) than in the HIGH category $(60 \%$ of the total 20,679 parasites counted) (Chi square value $=906, \mathrm{df}=1, p<0.05$ ). This indicates that gametogenesis and/or fertilization was a less frequent event in mosquitoes fed on the LOW category volunteers than in mosquitoes fed on HIGH category volunteers. Whether the lower frequency of round stages in the LOW category was due to poor gametogenesis or poor fertilization is unknown because 1) early development is not synchronous, and 2) it has never been firmly established that the monoclonal antibody used to detect pre-oocyst stages (i.e., Pvs25) can unequivocally differentiate round stages as either unfertilized female gametes or zygotes.

Early sporogony - parasite mortality and cohort life tables The average total parasite mortality $(K)$ from macrogametocyte to oocyst for all infections and mosquito species combined was 1.83 (antilog $=68$-fold or $98 \%$ loss) and ranged from 0.77 (6-fold or 83\% loss) to 3.36 (2,291-fold or $>99.9 \%$ loss) (Table 3 ). There was no overall effect on $K$ due to the mosquito species in which parasites developed (ANOVA, $\mathrm{F}=0.06, \mathrm{df}=2,24, \mathrm{p}=0.94$ ), but there was a significant effect on $K$ due to the particular volunteer from which the parasites originated (ANOVA, $\mathrm{F}=4.65$, $\mathrm{df}$ $=14,24, \mathrm{p}=0.01)$. The average $k-1$ mortality for all infections combined was 1.27 (i.e., 19-fold or 95\% loss) and ranged from 0.35 (2-fold or 55\% loss) to 2.48 (302-fold or $99.7 \%$ loss). There was no significant effect on $k-1$ due to mosquito species (ANOVA, $\mathrm{F}=1.97, \mathrm{df}=2,27, \mathrm{p}=$ $0.16)$, but there was a significant effect due to volunteer (ANOVA, $\mathrm{F}=5.16, \mathrm{df}=12,27, \mathrm{p}=0.002$ ). The average $k$ 2 mortality for all infections combined was 0.38 (i.e., 2 fold or $57 \%$ loss) and ranged from 0 (no loss) to 1.96 ( 91 fold or $99 \%$ loss). There were no significant effects on $k-2$ due to either mosquito species (Kruskal-Wallis ANOVA, F $=0.06, \mathrm{df}=2,30, \mathrm{p}=0.94$ ) or volunteer (Kruskal-Wallis ANOVA, $\mathrm{F}=1.84, \mathrm{df}=14,30, \mathrm{p}=0.12$ ). The average $k-3$ mortality for all infections combined was 0.35 (i.e., 2 -fold or $55 \%$ loss) and ranged from 0 (no loss) to 1.40 (25-fold or $96 \%$ loss). There were no significant effects on $k-3$ mortalities due to either mosquito species (Kruskal-Wallis ANOVA, $\mathrm{F}=0.83, \mathrm{df}=2,24, \mathrm{p}=0.45$ ) or volunteer (Kruskal-Wallis ANOVA, $\mathrm{F}=1.89, \mathrm{df}=14,24, \mathrm{p}=0.16$ ). 
Table I: Relative frequencies of Plasmodium vivax life-stages developing over 48 hours within Anopheles mosquitoes. Data for A. dirus, A. minimus, and $A$. sawadwongporni have been pooled.

\begin{tabular}{cccccc}
\hline TIME $(\mathrm{hr})$ & Round stage & Stage II Ookinete & Stage III Ookinete & Stage IV-VI Ookinete & Total Number of Parasites \\
\hline 12 & 0.81 & 0.11 & 0.05 & 0.03 & 1,116 \\
18 & 0.58 & 0.06 & 0.02 & 0.34 & 811 \\
20 & 0.62 & 0.07 & 0.03 & 0.28 & 2,006 \\
22 & 0.49 & 0.08 & 0.03 & 0.40 & 5,621 \\
24 & 0.46 & 0.09 & 0.03 & 0.42 & 3,156 \\
26 & 0.62 & 0.10 & 0.05 & 0.23 & 0.35 \\
36 & 0.48 & 0.09 & 0.08 & 0.38 & 2,138 \\
48 & 0.46 & 0.09 & 0.07 & & 498 \\
\hline
\end{tabular}

Different volunteers had a highly significant effect on infection outcome and not all mosquito species fed on all the volunteers, so it was appropriate that only co-infections were used to compare population dynamics of sporogony among mosquito species. There were seven coinfections of $A$. dirus and A. minimus (Volunteers 7, 8, 9, $12,13,14,15$ ) having complete life tables, but only three co-infections with A. sawadwongporni having complete life tables (Volunteers 12, 14, 15) - too few to provide meaningful analyses of $k-2$ and $K$ mortalities among A. sawadwongporni co-infections. Macrogametocyte densities were significantly greater in A. dirus than in co-infected A. minimus (geometric means $=190$ and 75, respectively; Wilcoxon signed rank test, $\mathrm{p}=0.008$ ), presumably because $A$. dirus ingested more blood than A. minimus (see above). There was no significant difference between co-infected $A$. dirus and A. minimus with respect to densities of round stages (means $=15$ and 14 , respectively; paired t-test $\mathrm{t}=$ $0.09, \mathrm{df}=6, \mathrm{p}=0.93$ ) or ookinetes (means $=9$ and 5 , respectively; paired t-test $\mathrm{t}=1.29, \mathrm{df}=6, \mathrm{p}=0.24$ ) but there was a significant difference with respect to oocyst densities (geometric means $=5$ and 1 , respectively; Wilcoxon signed rank test, $\mathrm{p}=0.03$ ). Although total parasite mortality did not differ significantly between co-infected A. dirus and A. minimus $(K=1.81$ and 1.76 , respectively; paired t-test $\mathrm{t}=0.35, \mathrm{df}=6, \mathrm{p}=0.74)$, the partitioned mortalities indicated that population processes differed between P. vivax developing within the two mosquito species. Macrogametocyte mortality was significantly greater in A. dirus than in co-infected $A$. minimus (average $k-1$ 's = 1.38 and 0.93 , respectively; paired t-test $\mathrm{t}=5.32, \mathrm{df}=6, \mathrm{p}$ $=0.003$ ). There was no significant difference in the mortality of round stages in either A. dirus or A. minimus (average $k-2$ 's $=0.47$ and 0.43 , respectively; paired t-test, $\mathrm{t}=$ $0.25, \mathrm{df}=6 ; \mathrm{p}=0.81$ ). However, ookinete mortality was significantly less in A. dirus than in A. minimus (average $k$ 3 's $=0.15$ and 0.40 , respectively; paired t-test, $\mathrm{t}=3.06, \mathrm{df}$ $=6 ; \mathrm{p}=0.02)$. Thus, the patterns of $P$. vivax stage-specific mortality differed between co-infected A. dirus and A. minimus. Within $A$. dirus, $k-1$ mortality was significantly greater than either $k-2$ and $k$-3 mortalities (ANOVA with Tukey's post-hoc test, $\mathrm{F}=14.8, \mathrm{df}=2,19, \mathrm{p}=0.0002$ ), indicative of Type III survivorship [30]; whereas in A. minimus there were no significant differences between $k-1$ or $k-2$ or $k-3$ mortalities (ANOVA, $\mathrm{F}=3.10, \mathrm{df}=2,18, \mathrm{p}=$ $0.069)$, indicative of Type II survivorship.

\section{Early sporogony - life-stage correlations}

For each density relationship examined (e.g., ookinete vs. oocyst densities, etc.) there were no significant differences among the 3 mosquito species with respect to the slopes or elevations of individual regression equations (analyses of covariance, $p<0.05[31]$ ). Therefore, data were pooled among mosquito species. There was no correlation

Table 2: Infection prevalences for Plasmodium vivax early sporogonic life-stages developing within Anopheles mosquitoes. The total numbers of mosquitoes examined per life-stage are given in parentheses. HIGH = volunteers who, at the time of donating blood, would be most likely to perpetuate $P$. vivax transmission in nature (i.e., yielding $\geq I$ oocyst per mosquito in $A$. dirus). LOW $=$ volunteer blood that would not be likely to perpetuate transmission in nature (i.e., yielding < I oocyst per mosquito in $A$. dirus).

\begin{tabular}{lcccc}
\hline Mosquito Species & Number of Volunteers & Round stage & Ookinete & Oocyst \\
\hline & HIGH & & & \\
A. dirus & 10 & $88.1 \%(637)$ & $78.6 \%(637)$ & $53.8 \%(513)$ \\
A. minimus & 5 & $89.3 \%(150)$ & $78.0 \%(150)$ & $56.4 \%(55)$ \\
A. sawadwongporni & 6 & $90.4 \%(167)$ & $81.4 \%(167)$ & $29.4 \%(34)$ \\
A. dirus & LOW & $21.7 \%(420)$ & $7.4 \%(420)$ & $3.2 \%(910)$ \\
A. minimus & 5 & $28.2 \%(252)$ & $12.7 \%(252)$ & $2.8 \%(71)$ \\
A. sawadwongporni & 4 & $3.4 \%(29)$ & $0.0 \%(29)$ & Not determined
\end{tabular}


Table 3: Cohort life tables for Plasmodium vivax developing within Anopheles mosquitoes. Stage-specific densities and mortality coefficients (k-values) for each cohort are given, where:k-I $=\log _{10}($ macrogametocyte $)-\log _{10}\left(\right.$ round stage);k-2 $=\log _{10}($ round stage $)$ $\log _{10}\left(\right.$ ookinete); $k-3=\log _{10}($ ookinete $)-\log _{10}($ oocyst $) ; K=k-1+k-2+k-3$. Values in parentheses $=$ antilogs of averaged $k$-values. For each Anopheles species, parasite values are ranked in descending order of macrogametocyte densities within the HIGH and LOW categories of infection intensity.

\begin{tabular}{|c|c|c|c|c|c|c|c|c|c|}
\hline Vol. & Class & Macrogametocyte & $k-I$ & Round stage & $k-2$ & Ookinete & $k-3$ & Oocyst & $K$ \\
\hline \multicolumn{10}{|c|}{ Anopheles dirus } \\
\hline 11 & $\mathrm{HIGH}$ & 2978.0 & $*$ & 3.23 & 1.96 & 32.69 & 1.40 & 1.30 & 3.36 \\
\hline 15 & & 1242.0 & 1.63 & 29.27 & 0.00 & 31.37 & 0.00 & 59.50 & 1.63 \\
\hline 2 & & 812.9 & 1.12 & 61.05 & 0.21 & 37.69 & 0.35 & 17.00 & 1.68 \\
\hline 5 & & 416.3 & $*$ & 42.39 & 0.33 & 192.58 & 0.65 & 42.70 & 0.98 \\
\hline 6 & & 358.6 & 1.38 & 15.08 & 0.17 & 10.15 & 0.63 & 2.40 & 2.18 \\
\hline 1 & & 332.8 & 1.44 & 12.20 & 0.00 & 13.43 & 0.00 & 25.10 & $\mathrm{I} .44$ \\
\hline 13 & & 277.7 & 0.85 & 39.33 & 0.52 & 11.88 & 0.77 & 2.00 & 2.14 \\
\hline 3 & & 233.3 & 1.37 & 10.02 & 0.00 & 14.03 & 0.00 & 85.80 & 1.37 \\
\hline 14 & & 96.1 & $*$ & 6.71 & 0.77 & 16.20 & 0.00 & 61.70 & 0.77 \\
\hline 12 & & 56.6 & 1.20 & 3.54 & 0.08 & 2.96 & 0.00 & 10.60 & 1.28 \\
\hline 8 & LOW & 804.4 & 2.15 & 5.70 & 0.77 & 0.15 & 0.06 & 0.00 & 2.98 \\
\hline 7 & & 165.5 & 0.93 & 19.63 & 1.17 & 1.32 & 0.25 & 0.30 & 2.35 \\
\hline 10 & & 138.8 & 2.06 & 0.23 & 0.09 & 0.00 & 0.00 & 0.10 & 2.15 \\
\hline 4 & & 101.8 & 1.58 & 2.70 & 0.34 & 1.23 & 0.14 & 0.60 & 2.06 \\
\hline 9 & & 34.7 & 1.54 & 0.00 & 0.00 & 0.00 & 0.00 & 0.00 & 1.54 \\
\hline \multicolumn{2}{|c|}{ Average Losses } & \multicolumn{2}{|c|}{ I.44 (27-fold) } & \multicolumn{2}{|c|}{0.43 (3-fold) } & \multicolumn{2}{|c|}{0.28 (2-fold) } & \multicolumn{2}{|c|}{ I.86 (72-fold) } \\
\hline \multicolumn{10}{|c|}{ Anopheles minimus } \\
\hline 15 & $\mathrm{HIGH}$ & 485.9 & 1.57 & 12.96 & 0.18 & 8.57 & 0.00 & 10.7 & 1.75 \\
\hline 2 & & 318.0 & 0.97 & 33.91 & 0.34 & 15.48 & ND & ND† & ND \\
\hline 13 & & 108.6 & 0.43 & 40.54 & 0.67 & 8.66 & 1.34 & 0.4 & 2.44 \\
\hline 14 & & 37.6 & 0.67 & 8.06 & 0.00 & 11.17 & 0.40 & 4.4 & 1.07 \\
\hline 12 & & 22.2 & 0.83 & 3.32 & 0.52 & 1.00 & 0.19 & 0.3 & 1.54 \\
\hline 8 & LOW & 314.7 & 1.60 & 7.89 & 0.70 & 0.76 & 0.25 & 0 & 2.55 \\
\hline 7 & & 64.8 & 0.35 & 29.18 & 0.83 & 4.32 & 0.64 & 0 & 1.82 \\
\hline 10 & & 54.3 & 1.73 & 0.00 & 0.00 & 0.00 & ND & ND & ND \\
\hline 9 & & 13.6 & 1.03 & 0.37 & 0.14 & 0.00 & 0.00 & 0 & 1.17 \\
\hline \multicolumn{2}{|c|}{ Average Losses } & \multicolumn{2}{|c|}{1.02 (10 -fold) } & \multicolumn{2}{|c|}{0.33 (2-fold) } & \multicolumn{2}{|c|}{0.40 (2-fold) } & \multicolumn{2}{|c|}{ I.76 (58-fold) } \\
\hline \multicolumn{10}{|c|}{ Anopheles sawadwongporni } \\
\hline 15 & $\mathrm{HIGH}$ & 518.3 & 1.51 & 15.92 & 0.34 & 7.26 & 0.58 & 1.9 & 2.43 \\
\hline 2 & & 339.2 & 1.00 & 34.23 & 0.54 & 9.98 & ND & ND & ND \\
\hline 13 & & 115.9 & 1.35 & 5.13 & 0.56 & 1.42 & ND & ND & ND \\
\hline 3 & & 97.4 & 1.08 & 8.08 & 0.24 & 4.67 & ND & ND & ND \\
\hline 14 & & 40.1 & 0.67 & 8.61 & 0.04 & 7.82 & 1.05 & 0.7 & 1.76 \\
\hline 12 & & 23.6 & 1.15 & 0.76 & 0.18 & 0.15 & 0.06 & 0 & 1.39 \\
\hline 8 & LOW & 335.7 & 2.48 & 0.12 & 0.05 & 0.00 & ND - & ND & ND \\
\hline$A v$ & osses & 1.27 (19-fo & & $0.38(2-$ & & 0.35 & & 1.83 & \\
\hline
\end{tabular}

* Not calculated because round stages were obviously under-sampled (note corresponding ookinete densities). Corresponding k-2 mortalities were therefore calculated as $\log _{10}$ (macrogametocyte) - $\log _{10}$ (ookinete).

$\dagger \mathrm{ND}=$ not determined due to insufficient numbers of mosquitoes available. 
between macrogametocyte density and oocyst prevalence $\left(\mathrm{r}^{2}=0.11, \mathrm{~F}=2.9, \mathrm{df}=1,24, \mathrm{p}=0.10\right)$, indicating that oocyst infection prevalence could not be predicted based on examination of a blood smear. However, the densities of successive life-stages were all significantly correlated i.e., macrogametocyte and round stage densities (Figure $\left.3 \mathrm{~A}, \mathrm{r}^{2}=0.23, \mathrm{~F}=8.3, \mathrm{df}=1,30, \mathrm{p}=0.007\right)$, round stage and ookinete densities (Figure $3 \mathrm{~B}, \mathrm{r}^{2}=0.58, \mathrm{~F}=39.6, \mathrm{df}=$ $1,30, \mathrm{p}<0.0001$ ), and ookinete and oocyst densities (Figure $\left.3 \mathrm{C}, \mathrm{r}^{2}=0.55, \mathrm{~F}=28.0, \mathrm{df}=1,24, \mathrm{p}<0.0001\right)$. Likewise, the correlations between life-stage densities and the stagespecific mortalities ( $k$ 's) acting on those life-stages were also statistically significant (or nearly so) - i.e., macrogametocyte density and $k-1$ (Figure $3 \mathrm{D}, \mathrm{r}^{2}=0.28, \mathrm{~F}=11.3$, $\mathrm{df}=1,30, \mathrm{p}=0.002$ ), round stage density and $k$-2 (Figure $\left.3 \mathrm{E}, \mathrm{r}^{2}=0.12, \mathrm{~F}=4.1, \mathrm{df}=1,30, \mathrm{p}=0.053\right)$, ookinete density and $k-3$ (Figure $3 \mathrm{~F}, \mathrm{r}^{2}=0.18, \mathrm{~F}=5.0, \mathrm{df}=1,24, \mathrm{p}=$ $0.036)$. A significant positive relationship between lifestage density and its corresponding $k$-value is suggestive of density-dependant mortality. However these variables are not independent of one another and, therefore, suspected density dependence could be spurious due to sampling errors [26]. To validate density dependence, the regression equations for successive life-stage densities (Figure 3A-C)
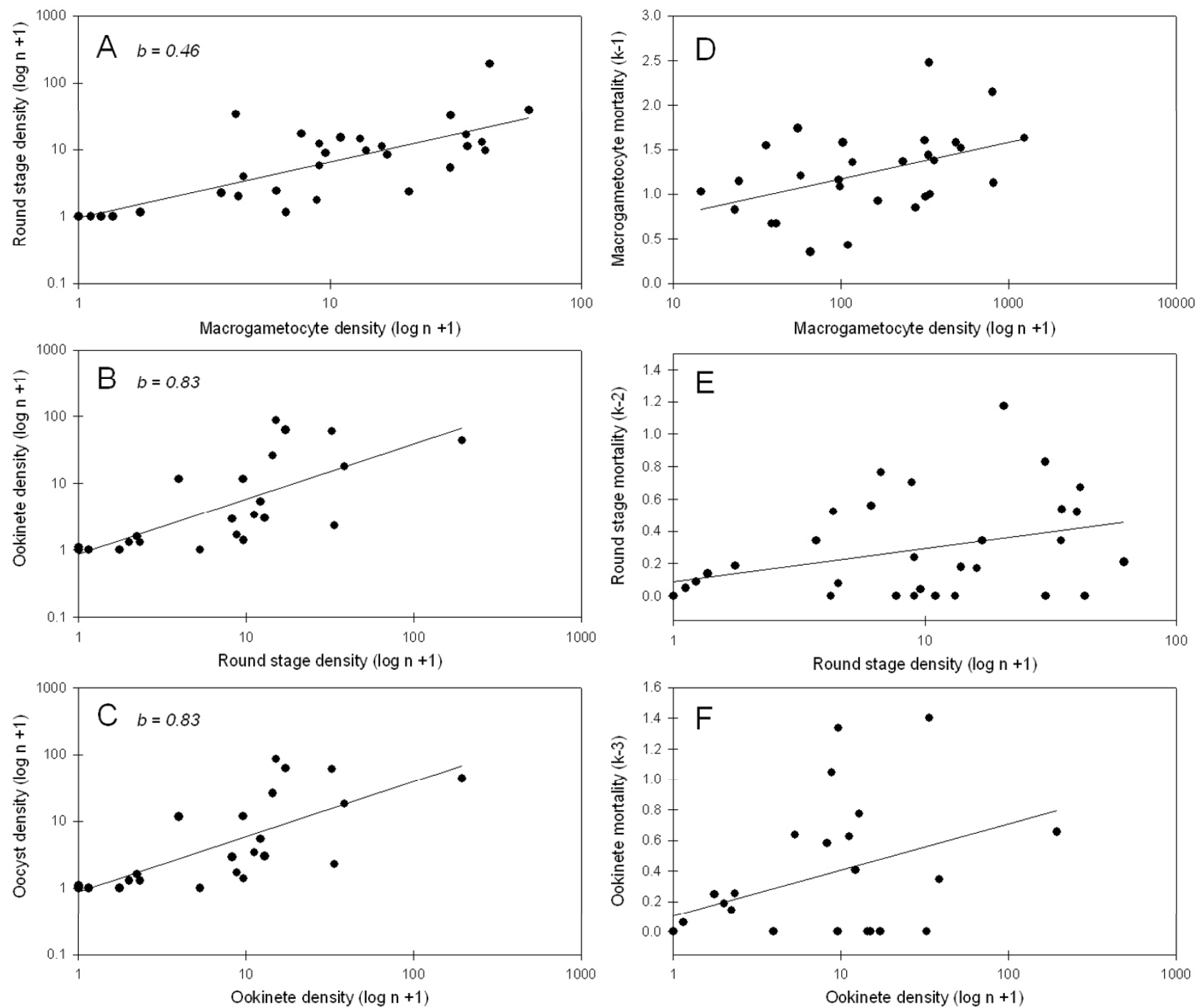

\section{Figure 3}

Density relationships during Plasmodium vivax early sporogony in Anopheles mosquitoes. Panels A, B, C illustrate density relationships between successive life-stages. Panel D, E, F illustrate density relationship between a life-stage and its corresponding mortality. Data for $A$. dirus, $A$. minimus and $A$. sawadwongporni mosquitoes were pooled because the regression equations for each of these relationships did not differ among mosquito species. 
were re-examined to test whether regression slopes departed significantly from 1.0 [32], which would confirm that density dependence is real [26]. The only density relationship whose slope departed significantly from 1.0 was that of macrogametocyte and round stage densities (Figure 3A, slope $=0.46$, t-test, $\mathrm{t}=2.04, \mathrm{df}=29, \mathrm{p}<0.05$ ) This indicates that density dependent mortality occurred during the macrogametocyte to round stage transition (i.e., gametogenesis/fertilization) but not during other life-stage transitions of early sporogony.

\section{Early sporogony - parasite distribution}

Patterns of parasite distribution within A. dirus, A. minimus and A. sawadwongporni were described by calculating Green's index of dispersion for round stages, ookinetes and oocysts in each infection where more than one infected mosquito was found for each of these life-stages (Figure 4). Mean dispersion indices for round stages (0.0102) and ookinetes (0.0119) did not differ significantly from one another (paired t-test, $\mathrm{t}=0.60, \mathrm{df}=17, \mathrm{p}$ $=0.56$ ) but both indices were significantly lower than mean dispersion indices for oocysts (0.0984) (paired ttests, t values $>3.0, \mathrm{df}=17$, $\mathrm{p}$ values $=0.006$ ). Round stages and ookinetes developed within mosquitoes in a more homogenous manner and were more randomly distributed among individual mosquitoes than were oocysts regardless of parasite density, fertilization success or parasite origin (HIGH versus LOW volunteer).

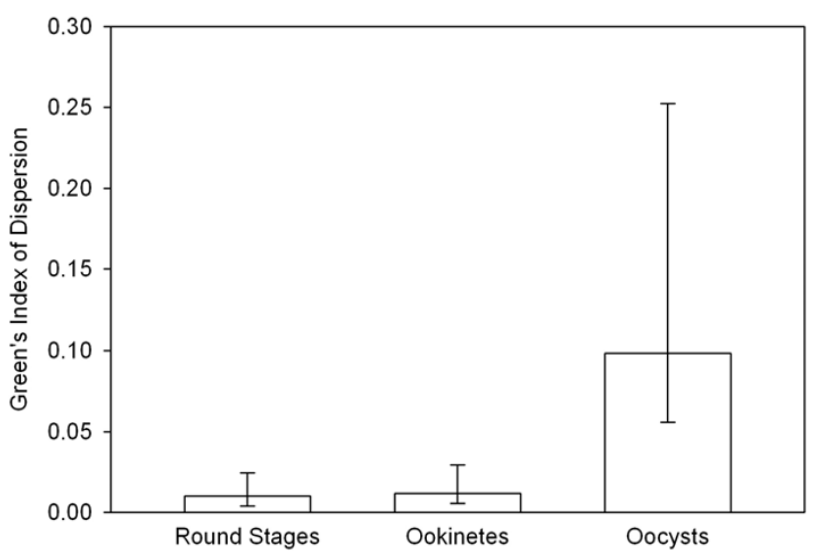

\section{Figure 4}

Dispersal indices for Plasmodium vivax life-stages developing within Anopheles dirus, A. minimus and A. sawadwongporni mosquitoes. Bars indicate the mean indices for each parasite life-stage. Error bars indicate $95 \%$ confidence limits. A Green's index of zero indicates that parasites were randomly distributed among mosquitoes. Higher values indicate that parasites were more unevenly distributed among mosquitoes, suggesting that there was greater heterogeneity among individual mosquitoes in their susceptibility for that specific parasite life-stage.
Late sporogony - kinetics of oocyst growth, sporozoite release and invasion into mosquito salivary glands

All five volunteers providing parasites for this phase of the study were infected with the VK210 strain of $P$. vivax. There was no significant difference in the diameters of oocysts developing within $A$. dirus versus $A$. minimus (ttest, $\mathrm{t}=-0.01, \mathrm{~d}=316, \mathrm{p}=0.99)$. Mean oocyst diameters did not increase significantly from days 7-10, but increased almost 2-fold from days 12 to 14 . Thereafter, oocyst size stabilized and mean oocyst diameters did not change significantly throughout the remainder of the study (Table 4). The rapid swelling of oocysts at days 1214 corresponded to sporozoite release (Figure 5). Oocyst release of sporozoites into the haemocoel occurred as early as day 12 for A. dirus and day 14 for A. minimus. Sporozoite invasion into the salivary glands occurred at day 14 for both species. This indicated that sporozoite invasion into the glands of $A$. dirus may have been a somewhat more protracted process requiring one to two days, whereas in A. minimus, sporozoites invaded the salivary glands within one day after being released from the oocyst. The kinetics of sporozoite release and invasion into the salivary glands were similar for each mosquito species regardless of whether the infection was of high or low intensity (Figure 5, Volunteers 16 and 18, respectively). However, not all oocysts released sporozoites. Even after massive release of sporozoites into the haemocoel on days 12 and 14, there remained a substantial number of fully-formed oocysts on the gut that had failed to release sporozoites by day 22 (Figure 5, Volunteers 17, 19 and 20).

\section{Late sporogony - sporozoite production and invasion efficiency into mosquito salivary glands}

The per capita production of sporozoites was calculated for each infection using mean oocyst densities from mosquitoes sampled on days 7 to 10 prior to the release of sporozoites. Mean oocyst densities ranged from two to 131 oocysts per mosquito (Table 5). Total sporozoite production was calculated using mosquitoes sampled on days 12 to 18 and included all infected mosquitoes, even those that were oocyst-positive but sporozoite-negative (i.e., to account for possible abortive infections). Mean per capita production of sporozoites ranged from 169 to 784 sporozoites per oocyst. There was no significant difference in sporozoite production between oocysts developing within $A$. dirus versus those within $A$. minimus ( $\mathrm{t}-$ test, $\mathrm{t}=-1.87, \mathrm{~d}=5, \mathrm{p}=0.12$ ). The overall average for both mosquito species combined was $508 \pm 230$ sporozoites per oocyst. Only sporozoite-positive mosquitoes were used to calculate the efficiency of sporozoite invasion into the salivary glands. Sporozoite invasion into salivary glands was consistently efficient from infection to infection, with no significant difference between sporozoites developing within $A$. dirus versus $A$. minimus (t-test, $\mathrm{t}=$ 
Table 4: Plasmodium vivax oocyst growth in Anopheles dirus and A. minimus mosquitoes during late sporogony. Values indicate mean oocyst diameters $(\mu \mathrm{m})$ with $\mathbf{9 5} \%$ confidence intervals in parentheses and accompanying letters signifying statistically significant differences in mean diameters.

\begin{tabular}{|c|c|c|c|c|c|c|c|c|c|}
\hline Day & 7 & 9 & 10 & 12 & 14 & 16 & 18 & 20 & 22 \\
\hline Oocyst Diameter $(\mu \mathrm{m})$ & $\begin{array}{c}27 c \\
(22-32)\end{array}$ & $\begin{array}{c}31 \mathrm{c} \\
(27-35)\end{array}$ & $\begin{array}{c}34 c \\
(3 I-36)\end{array}$ & $\begin{array}{c}49 \mathrm{~b} \\
(46-5 \mathrm{I})\end{array}$ & $\begin{array}{c}58 \mathrm{a} \\
(55-6 \mathrm{I})\end{array}$ & $\begin{array}{c}58 \mathrm{a} \\
(55-62)\end{array}$ & $\begin{array}{c}55 \mathrm{ab} \\
(52-58)\end{array}$ & $\begin{array}{c}53 \mathrm{ab} \\
(46-60)\end{array}$ & $\begin{array}{c}52 \mathrm{ab} \\
(42-63)\end{array}$ \\
\hline No. Mosq. & 13 & 12 & 28 & 58 & 64 & 57 & 47 & 24 & 15 \\
\hline
\end{tabular}

*Different letters indicate significant differences in oocyst diameter (Tukey post-hoc test, $p<0.05$ ).

$0.46, \mathrm{~d}=5, \mathrm{p}=0.66)$. The overall proportion of haemocoel sporozoites that succeeded in invading the salivary glands was estimated to be $0.737 \pm 0.099$, or roughly three-quarters of the total sporozoites produced.

\section{Late sporogony - life-stage correlations}

There were no differences in regression equations between A. dirus and A. minimus (analyses of covariance, p's $>0.05$ ) and therefore data were pooled. There was a significant correlation between the average oocyst density present on midguts prior to sporozoite release and the subsequent production of sporozoites (Figure $6 \mathrm{~A} ; \mathrm{r}^{2}=0.86, \mathrm{~F}=31.9$, $\mathrm{df}=1,6, \mathrm{p}=0.002$ ). Likewise, there was a highly significant correlation between average sporozoite production and sporozoite density in the salivary glands (Figure 6B; $\mathrm{r}^{2}=$ 0.99, $\mathrm{F}=1226.6, \mathrm{df}=1,6, \mathrm{p}<0.0001)$.

\section{Discussion}

It has long been known that some gametocyte carriers are more infectious to mosquitoes and produce more oocysts than do other gametocyte carriers [33-35]. This was also the case in the present study. On average, $P$. vivax populations experienced a 68 -fold loss in abundance from macrogametocyte to oocyst and there were no differences in the magnitude of overall parasite losses among the 3 mosquito species tested ( $K$, Table 3 ). However, losses within individual cohort infections varied tremendously (6-fold to over 2,000-fold) depending on the source of the gametocyte population (i.e., the volunteer). Life tables showed clearly that mortality during gametogenesis and fertilization (i.e., $k-1$ ) was generally the most critical transition determining an infection outcome (Table 3 ). Importantly, differences among volunteers in gametocyte and trophozoite densities, gametocyte sex ratios and blood haematocrits did not correlate with differences in volunteer infectiousness - an observation noted previously by other workers [36-38]. Obviously, something else influenced the early developmental success rates of different gametocyte populations. In the broad sense, possibilities may include factors intrinsic to the parasite (e.g., gametocyte immaturity or senescence) or extrinsic factors contained within the blood (e.g., antibody, cytokines, drugs, etc.). However, it seems unlikely that mosquito factors contributed to the high infection-to-infection variability observed in $k-1$ (macrogametocyte) or $k-2$ (round stage) mortalities because the dispersion indices for round stages and ookinetes were essentially zero (Figure 4). This means that within almost every infection, the processes affecting production of round stages and ookinetes occurred randomly among mosquitoes, with little mosquito-to-mosquito heterogeneity - regardless of whether a particular infection was successful or not. Furthermore, significant variability in $k-1$ mortalities was observed among volunteers but not among mosquito species. Thus, blood and/ or parasite factors exerted a more dominant influence on $k-1$ and $k-2$ mortalities than did mosquito factors.

One source of $k-1$ mortality may be inferred by examining differences among individual infections in life-stage prevalence (Table 2). In this study, all unfed mosquitoes were removed at the onset of each infection so that in theory the starting gametocyte prevalence was virtually $100 \%$. However by 12 to $48 \mathrm{~h}$ after ingesting gametocytes, a significantly lower prevalence of round stages was observed in mosquitoes fed blood from poorly infectious people (LOW; Table 2) than in mosquitoes fed blood from more infectious people (HIGH). This marked decline in the prevalence of round stages suggests that gametogenesis and/or fertilization was sub-optimal in blood from poorly infectious people. In those mosquitoes that did produce round stages when fed poorly infectious blood, the ratio of round stages to mature ookinetes was significantly higher than in mosquitoes fed more infectious blood. Presumably, a higher ratio of round stages-to-ookinetes persisting throughout the 2 day sampling period means that round stages produced from the poorly infectious volunteers were less likely to complete their development to ookinetes. Thus, $P$. vivax gametocytes within poorly infectious people displayed sub-optimal fertilization and/or zygote differentiation. Another intriguing, albeit minor, source of $k-1$ mortality was that of density-dependant mortality of macrogametocytes - i.e., the per capita conversion of gametocytes to round stages decreased with increasing gametocyte density (Figures $3 \mathrm{~A}$ and $3 \mathrm{D}$ ). The mechanisms underlying this form of population regulation is speculative but one plausible explanation is polyspermy - i.e., simultaneous fertilization of a single female gamete with more than one male gamete. As the 


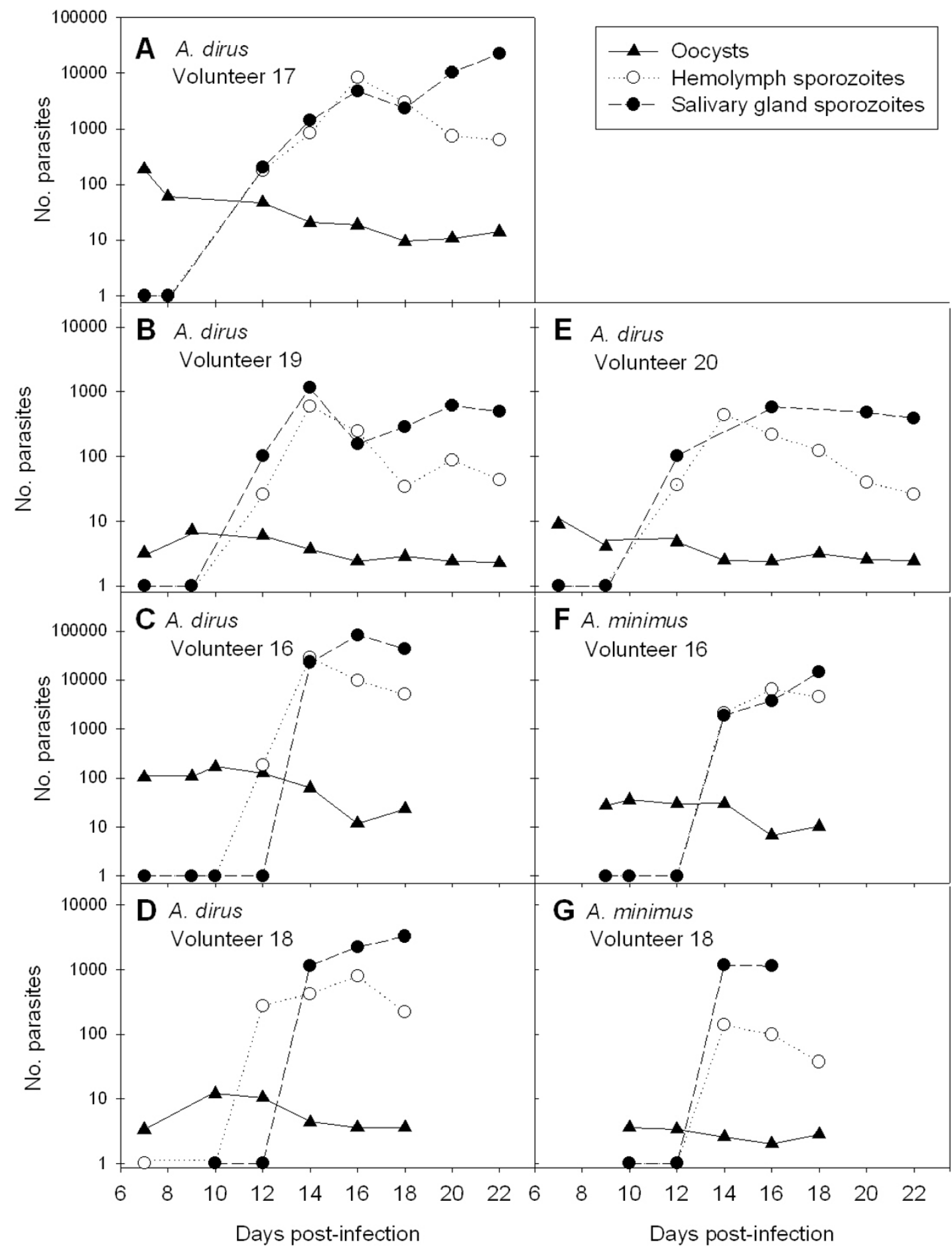

Figure 5

Developmental kinetics of Plasmodium vivax sporozoite release from oocysts and invasion into mosquito salivary glands. Parasite densities ( $n+I$; shown on log scale) were recorded for Anopheles dirus (Panels $A-E)$ and $A$. minimus (Panels F-G) from Days 7 to 22 post-infection (p.i.). Results for co-infections of $A$. dirus and A. minimus are shown for Volunteers 16 (Panels $C$ and F) and 18 (Panels D and G); data were collected only until Day 18 p.i. 
Table 5: Per capita production of Plasmodium vivax sporozoites and subsequent invasion efficiency into mosquito salivary glands.

\begin{tabular}{|c|c|c|c|}
\hline Volunteer & $\begin{array}{l}\text { Geometric Mean Oocyst Density (days } \\
\qquad 7-12 \text { ) }\end{array}$ & $\begin{array}{l}\text { Sporozoites Produced per Oocyst (days } \\
\qquad 12-18 \text { ) }\end{array}$ & $\begin{array}{l}\text { Proportion of Sporozoites Invading the } \\
\text { Salivary Glands (days I2-18) }\end{array}$ \\
\hline & \multicolumn{3}{|c|}{ Anopheles dirus } \\
\hline 17 & 42 & 310 & 0.613 \\
\hline 18 & 5 & 668 & 0.791 \\
\hline 19 & 2 & 350 & 0.742 \\
\hline 20 & 3 & 169 & 0.840 \\
\hline \multirow[t]{2}{*}{ Average \pm SD } & & $422 \pm 212$ & $0.749 \pm 0.085$ \\
\hline & \multicolumn{3}{|c|}{ Anopheles minimus } \\
\hline 16 & 30 & 664 & 0.590 \\
\hline 18 & 2 & 784 & 0.826 \\
\hline Average \pm SD & & $724 \pm 85$ & $0.708 \pm 0.167$ \\
\hline
\end{tabular}

Overall average sporozoite production for both mosquito species combined $=508 \pm 230$ sporozoite/oocyst. Overall proportion of sporozoites entering salivary glands $=0.737 \pm 0.099$.

density of gametocytes increases, the likelihood of polyspermy would also increase. Polyspermy is lethal to the zygotes of most organisms [39] but it has only been described in eukaryotic organisms. Density dependent mortality was not confirmed for later life-stages.

Overall, ookinete mortality was low (2-fold loss). Unlike that of gametocytes and round stages, mortality of ookinetes $(k-3)$ was probably more strongly influenced by mosquito factors. In paired comparisons, dispersion indices for round stages and ookinetes were significantly lower than indices for oocysts (Figure 4). Thus, parasites within the blood meal were initially distributed randomly among mosquitoes but became more aggregated as ookinetes exited the midgut to form oocysts. The implication is that there was heterogeneity with respect to an individual mosquito's permissiveness to ookinete penetration and establishment on the outer midgut wall. Some mosquitoes simply presented a more hostile environment than others. Mosquito factors that act as mortality factors to block the ookinete conversion to oocyst include peritrophic matrix [40-42], digestive enzymes [43-45] and an array of immune effectors produced in response to ookinete invasion [reviewed in [46-48]]. It may be expected that there is some degree of variation in the vigor and timing of these processes among individual mosquitoes and that this variation, coupled with the individual variation in kinetics of ookinete formation (see Figure 2), produced the observed heterogeneity in oocyst densities. Interestingly, the mean dispersion indices for $P$. vivax oocysts recorded in this study using laboratory colonies of $A$. dirus $(0.092 \pm 0.138)$ and A. minimus $(0.082 \pm 0.093)$ mosquitoes were not statistically different from dispersion indices calculated from the published data of Rosenberg et al. [49] on P. vivax oocyst populations infecting wild-caught
A. dirus (0.114) and A. minimus (0.087) from southeastern Thailand ( $\mathrm{t}$-tests, $\mathrm{p}>0.58$ ). This supports the notion that the population dynamics of early sporogony described herein are similar to that which occurs in nature.

The classic view of sporozoite production is that each oocyst contains several thousand sporozoites. Indeed, meticulous studies where individual $P$. vivax oocysts were plucked from the midguts of $A$. dirus reported that each of 26 oocysts contained a mean of 3,688 sporozoites [50]. However, the present studyclearly indicates that not every oocyst achieves its full production potential (Table 5, Figure 5). Some oocysts probably contribute more sporozoites to the overall standing crop than others, whereas some oocysts may not contribute any sporozoites at all. The estimates of 169 to 784 sporozoites per oocyst reflect the per capita production of the entire oocyst population on a mosquito midgut and are reasonably similar to estimates obtained by linear regression of oocyst densities plotted against salivary gland sporozoites - i.e., 850 gland sporozoites per oocyst for P. vivax in A. dirus [38] and 663 gland sporozoites per oocyst for P. falciparum in A gambiae [12]. In the present study, apparently healthy oocysts were present on midguts for up to 22 days, i.e., more than 1 week after the initial surge of sporozoites into the haemocoel (Figure 5). Why some oocysts failed to release sporozoites is not known but of the 23,632 oocysts examined during the course of this study, no melanized oocysts were observed, suggesting that the mosquito melanization response was not responsible. If each $P$. vivax oocyst in $A$. dirus contains an average of 3,688 sporozoites [50] but the per capita production of the oocyst population averaged only 422 sporozoites per oocyst (Table 5 ), then only $11 \%$ of the potential sporozoite production and release was 

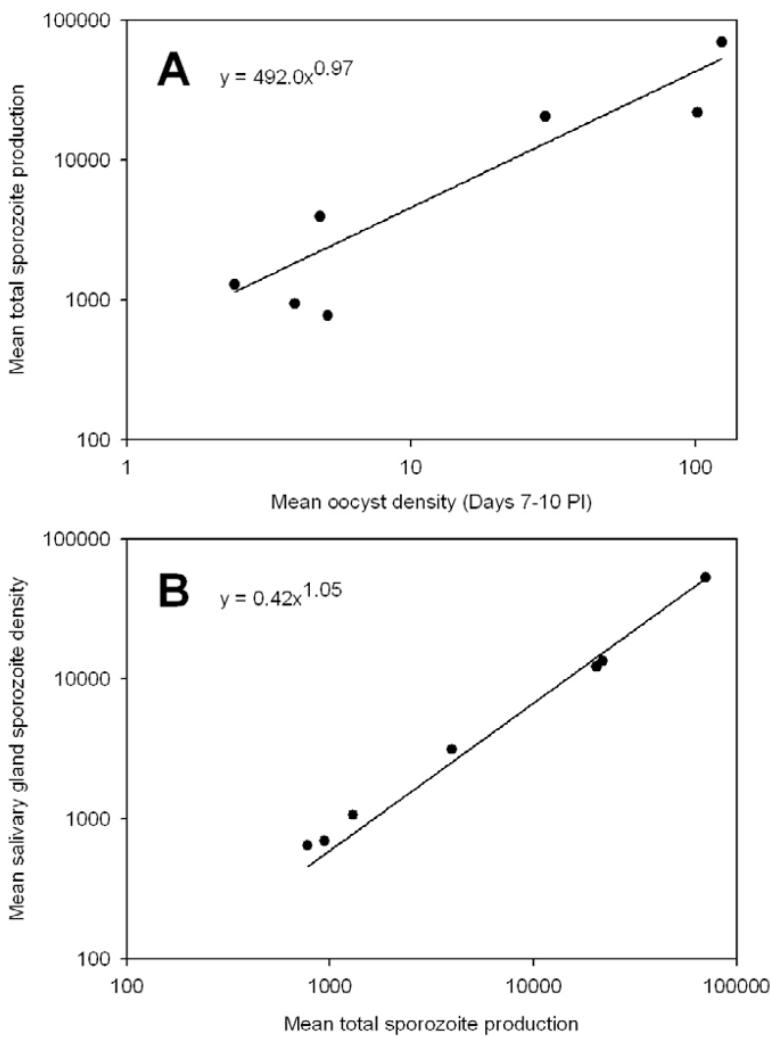

Figure 6

Density relationships of Plasmodium vivax during late sporogony in Anopheles dirus and A. minimus mosquitoes. A) Plasmodium vivax oocyst density (Days 7-10) and sporozoite production (Days I2-18) and B) sporozoite production and density of sporozoites in the mosquito salivary glands (Days 12-18).

actually realized by day 18 of infection. It remains to be determined whether laggard oocysts sequentially release fresh sporozoites into the haemocoel throughout the life time of their mosquito host or whether they simply stop producing sporozoites.

Even though initial oocyst release of sporozoites was inefficient, the invasion of $P$. vivax haemolymph sporozoites into the salivary glands of $A$. dirus and $A$. minimus was very efficient. Nearly $75 \%$ of all sporozoites produced successfully entered the glands (Table 5). These estimates are similar to efficiency estimates using similar methodology for $P$. falciparum sporozoite invasion into A. gambiae salivary glands $(=89 \%,[12])$. Total sporozoite production was related linearly to oocyst density and likewise, sporozoite density in the salivary glands was linearly related to the total sporozoite production of an infection (Figure 6). Thus within the intensity levels observed in this study, there was no obvious "saturation effect" of having too many oocysts on the gut or too many sporozoites in the haemolymph or salivary glands. This finding is compatible with findings from other Plasmodium/Anopheles systems $[12,51]$ and, when taken together, suggest that once ookinetes cross the midgut and establish themselves as oocysts, nutrients for further parasite population growth within mosquitoes are essentially unlimited and there is no "carrying capacity" imposed on developing oocysts by their "habitat".

\section{Conclusion}

This study describes the population dynamics of sporogony for 20 natural isolates of $P$. vivax from western Thailand in three species of colonized Anopheles species; $A$. dirus, A. minimus and A. sawadwongporni. Overall, there was a 68 -fold loss in abundance in parasite development from macrogametocyte to oocyst but the magnitude of parasite losses within individual infections ranged from 6 fold to over 2,000-fold. Gametogenesis and/or fertilization were the most critical processes determining the infection outcome. Subsequent parasite losses during round stage transformation and ookinete migration were generally less variable among infections. Indices of parasite dispersion suggested that parasites losses during fertilization and round stage transformation were more influenced by factors intrinsic to the parasite and/or factors within human blood, whereas losses during ookinete migration were more strongly influenced by mosquito factors. Sporozoite release from oocysts occurred on days 12 and 14 for A. dirus and A. minimus, respectively. Sporozoites in the haemocoel invaded the salivary glands efficiently (ca. 74\%) within a day or two. Not all oocysts produced sporozoites. Understanding population dynamics of sporogony in nature may help predict the efficacy of intervention strategies that target sporogony.

\section{Authors' contributions}

JV and RC designed the study. JS managed logistics and coordinated with the Thai Ministry of Public Health. GZ, NP, SP, GG and JV conducted the studies. GZ, JB, and JV performed statistical analyses and artwork. GZ and JV wrote the manuscript. All authors have read and approved the final manuscript.

\section{Acknowledgements}

The authors are grateful to Khun Peenuj Maneechai and other expert staff at the Department of Entomology, AFRIMS, and the staff of the Mae Sot Malaria Clinic (Thai Ministry of Public Health), for their support during this study. Monoclonal antibody to $P$. vivax sexual stage antigen, Pvs25, was kindly provided by Dr. Carole Long (National Institutes of Health). The work was performed while G. E. Zollner held a National Research Council Associateship Award at AFRIMS. The opinions or assertions contained within this manuscript are those of the authors and do not necessarily reflect the official views of the Department of Defense or the Armed Forces Research Institute of Medical Sciences. This project was funded by $\mathrm{NIH}$ grant Al488I 3 (JV). 


\section{References}

I. Beier JC: Malaria parasite development in mosquitoes. Ann Rev Entomol 1998, 43:519-543.

2. Sinden RE: Plasmodium differentiation in the mosquito. Parassitologia 1999, 41:139-148.

3. Kaslow DC: Transmission-blocking vaccines: uses and current status of development. Int J Parasitol I997, 27: I83-I89.

4. Carter R, Mendis KN, Miller LH, Molineaux L, Saul A: Malaria transmission-blocking vaccines - how can their development be supported? Nat Med 2000, 6:24I-244.

5. Tsuboi T, Tachibana M, Kaneko O, Torii M: Transmission-blocking vaccine of vivax malaria. Parasitol Int 2003, 52: I-I I.

6. James AA, Beerntsen BT, Capurro Mde L, Coates CJ, Coleman J, Jasinskiene N, Krettli AU: Controlling malaria transmission with genetically-engineered, Plasmodium-resistant mosquitoes: milestones in a model system. Parassitologia |999, 41:46 I-47|.

7. Jacobs-Lorena M: Interrupting malaria transmission by genetic manipulation of anopheline mosquitoes. J Vector Borne Dis 2003, 40:73-77.

8. Christophides GK: Transgenic mosquitoes and malaria transmission. Cell Microbiol 2005, 7:325-333.

9. Siden-Kiamos I, Louis C: Interactions between malaria parasites and their mosquito hosts in the midgut. Insect Biochem Mol Biol 2004, 34:679-685.

10. Baton LA, Ranford-Cartwright LC: Spreading the seeds of million-murdering death: metamorphoses of malaria in the mosquito. Trends Parasitol 2005, 2 I:573-580.

II. Vinetz JM: Plasmodium ookinete invasion of the mosquito midgut. Curr Top Microbiol Immunol 2005, 295:357-382

12. Vaughan JA, Noden BH, Beier JC: Population dynamics of Plasmodium falciparum in laboratory-infected Anopheles gambiae. J Parasit 1992, 78:716-724.

13. Vaughan JA, Hensley L, Beier JC: Sporogonic development of Plasmodium yoelii in five anopheline species. J Parasit 1994, 80:674-681.

14. Vaughan JA, Noden BH, Beier JC: Sporogonic development of cultured Plasmodium falciparum in six species of laboratoryreared Anopheles. Am J Trop Med Hyg 1994, 5 I:233-243.

15. Vaughan JA, Noden BH, Beier JC: Prior blood feeding effects on susceptibility of Anopheles gambiae (Diptera: Culicidae) to infection with cultured Plasmodium falciparum (Haemosporida: Plasmodiidae). J Med Entomol 1994, 3 I:445-449.

16. Alavi Y, Arai M, Mendoza J, Tufet-Bayona M, Sinha R, Fowler K, Billker O, Franke-Fayard B, Janse CJ, Waters A, Sinden RE: The dynamics of interactions between Plasmodium and the mosquito: $a$ study of the infectivity of Plasmodium berghei and Plasmodium gallinaceum, and their transmission by Anopheles stephensi, Anopheles gambiae and Aedes aegypti. Int J Parasit 2003, 33:933-943

17. Gouagna LC, Mulder B, Noubissi E, Tchuinkam T, Verhave JP, Boudin C: The early sporogonic cycle of Plasmodium falciparum 1998. in laboratory-infected Anopheles gambiae: an estimation of parasite efficacy. Trop Med Int Health 1998, 3:21-28.

18. Gouagna LC, Bonnet S, Gounoue R, Verhave JP, Eling W, Sauerwein $R$, Boudin C: Stage-specific effects of host plasma factors on the early sporogony of autologous Plasmodium falciparum isolates within Anopheles gambiae. Trop Med Int Health 2004, 9:937-948.

19. Okech BA, Gouagna LC, Walczak E, Kabiru EW, Beier JC, Yan G, Githure Il: The development of Plasmodium falciparum in experimentally infected Anopheles gambiae (Diptera: Culicidae) under ambient microhabitat temperature in western Kenya. Acta Trop 2004, 92:99-108.

20. Okech BA, Gouagna LC, Kabiru EW, Walczak E, Beier JC, Yan G, Githure Jl: Resistance of early midgut stages of natural Plasmodium falciparum parasites to high temperatures in experimentally infected Anopheles gambiae (Diptera: Culicidae). J Parasitol 2004, 90:764-768.

21. Gouagna LC, Ferguson HM, Okech BA, Killeen GF, Kabiru EW, Beier JC, Githure JI, Yan G: Plasmodium falciparum malaria disease manifestations in humans and transmission to Anopheles gambiae: a field study in Western Kenya. Parasitol 2004, I 28:235-243.

22. Chareonviriyaphap T, Bangs MJ, Ratanatham S: Status of malaria in Thailand. Southeast Asian J Trop Med Public Health 2000, 3 I :225-237.
23. Coleman RE, Sithiprasasna R, Kankaew P, Kiaattiut C, Ratanawong S, Khuntirat B, Sattabongkot J: Naturally occurring mixed infection of Plasmodium vivax VK2 10 and P. vivax VK247 in Anopheles mosquitoes (Diptera: Culicidae) in western Thailand. J Med Entomol 2002, 39:556-559.

24. Zollner GE, Ponsa N, Coleman RE, Sattabongkot J, Vaughan JA: Evaluation of procedures to determine absolute density of Plasmodium vivax ookinetes. J Parasitol 2005, 9 I:453-457.

25. Janse CJ, Mons B, Rouwenhorst RJ, Van der Klooster PF, Overdulve JP, Van der Kaay HJ: In vitro formation of ookinetes and functional maturity of Plasmodium berghei gametocytes. Parasitol 1985, 91:9-29.

26. Southwood TRE, Henderson PA: The construction, description, and analysis of age-specific life-tables. In Ecological Methods 3rd edition. Malden, MA: Blackwell Publishing; 2000:404-436.

27. Varley GC, Gradwell GR, Hassell MP: Insect population ecology: An analytical approach University of California Press; 1973.

28. Green $\mathrm{RH}$ : Measurement of non-randomness in spatial distributions. Res Pop Ecol 1966, 8: 1-7.

29. Ludwig JA, Reynolds JF: Statistical Ecology John Wiley \& Sons.; 1988.

30. Deevey ES: Life tables for natural populations of animals. Quart Rev Biol 1947, 22:283-3 I4.

31. Zar JH: Comparing simple linear regression equations. In Biostatistical Analysis 4th edition. Upper Saddle River, NJ: Prentice Hall; 1999:360-376.

32. Zar JH: Simple linear regression. In Biostatistical Analysis 4th edition. Upper Saddle River, NJ: Prentice Hall; 1999:324-359.

33. Darling ST: Factors in the transmission and prevention of malaria in the Panama Canal Zone. Ann Trop Med Parasitol 1910 4:179-223.

34. James SP: Some general results of a study of induced malaria in England. Trans Royal Soc Trop Med Hyg I931, 24:478-525.

35. Jeffery GM, Eyles DE: Infectivity of mosquitoes to Plasmodium falciparum as related to gametocyte density and duration of infectivity. Am J Trop Med Hyg 1955, 4:78I-789.

36. Kligler IJ, Mer G: Studies on the effect of various factors on the infection rate of Anopheles elutus with different species of Plasmodium. Ann Trop Med Parasitol 1937, 3 I:71-83.

37. Eyles DE, Young MD, Burgess RW: Studies on imported malarias 8. Infectivity to Anopheles quadrimaculatus of asymptomatic Plasmodium vivax parasitaemias. I Natl Malaria Soc 1948, 2:125-133.

38. Sattabongkot J, Maneechai N, Rosenberg R: Plasmodium vivax: gametocyte infectivity of naturally infected Thai adults. Parasitol 1991, I02:27-31.

39. Jaffee LA, Gould M: Polyspermy preventing mechanisms. In Biology of Fertilization Volume 3. New York, NY: Academic Press; 1985:223-250.

40. Sieber KP, Huber M, Kaslow D, Banks SM, Torii M, Aikawa M, Miller $\mathrm{LH}$ : The peritrophic membrane as a barrier: its penetration by Plasmodium gallinaceum and the effect of a monoclonal antibody to ookinetes. Exp Parasitol I991, 72:I45-156.

4I. Huber M, Cabib E, Miller LH: Malaria parasite chitinase and penetration of the mosquito peritrophic membrane. Proc Natl Acad Sci U S A 1991, 88:2807-2810.

42. Shahabuddin M: Plasmodium ookinete development in the mosquito midgut: a case of reciprocal manipulation. Parasitol 1998, I | 6:S83-93.

43. Gass RF: Influences of blood digestion on the development of Plasmodium gallinaceum (Brumpt) in the midgut of Aedes aegypti (L.). Acta Trop 1977, 34: I 27-I40.

44. Gass RF, Yeates RA: In vitro damage of cultured ookinetes of Plasmodium gallinaceum by digestive proteinases from susceptible Aedes aegypti. Acta Trop 1979, 36:243-52.

45. Feldmann AM, Billingsley PF, Savelkoul E: Bloodmeal digestion by strains of Anopheles stephensi Liston (Diptera: Culicidae) of differing susceptibility to Plasmodium falciparum. Parasitol 1990, 101:193-200.

46. Dimopoulos G: Insect immunity and its implication in mosquito-malaria interactions. Cell Microbiol 2003, 5:3-I4.

47. Kumar S, Barillas-Mury C: Ookinete-induced midgut peroxidases detonate the time bomb in anopheline mosquitoes. Insect Biochem Mol Biol 2005, 35:72 I-727.

48. Michel K, Kafatos FC: Mosquito immunity against Plasmodium. Insect Biochem Mol Biol 2005, 35:677-689. 
49. Rosenberg R, Andre RG, Somchit L: Highly efficient dry season transmission of malaria in Thailand. Trans $R$ Soc Trop Med Hyg 1990, 84:22-28.

50. Rosenberg R, Rungsiwongse J: The number of sporozoites produced by individual malaria oocysts. Am J Trop Med Hyg 1991, 45:574-577.

51. Rivero A, Ferguson HM: The energetic budget of Anopheles stephensi infected with Plasmodium chabaudi: is energy depletion a mechanism for virulence? Proc Royal Soc Lond B 2003, 270:|365-|37|.

Publish with Bio Med Central and every scientist can read your work free of charge

"BioMed Central will be the most significant development for disseminating the results of biomedical research in our lifetime. " Sir Paul Nurse, Cancer Research UK

Your research papers will be:

- available free of charge to the entire biomedical community

- peer reviewed and published immediately upon acceptance

- cited in PubMed and archived on PubMed Central

- yours - you keep the copyright
BioMedcentral 